Adam P. Vogel, PhD

Matthew L. Poole, MSc Hugh Pemberton, MSc

Marja W.J. Caverlé, MSc Frederique M.C.

Boonstra, MSc

Essie Low, DPsych

David Darby, MBBS,

$\mathrm{PhD}$

Amy Brodtmann, MBBS, $\mathrm{PhD}$

Correspondence to Dr. Vogel: vogela@unimelb.edu.au

Supplemental data at Neurology.org

\section{Motor speech signature of behavioral variant frontotemporal dementia}

\author{
Refining the phenotype
}

\section{ABSTRACT}

Objective: To provide a comprehensive description of motor speech function in behavioral variant frontotemporal dementia (bvFTD).

Methods: Forty-eight individuals (24 bvFTD and 24 age- and sex-matched healthy controls) provided speech samples. These varied in complexity and thus cognitive demand. Their language was assessed using the Progressive Aphasia Language Scale and verbal fluency tasks. Speech was analyzed perceptually to describe the nature of deficits and acoustically to quantify differences between patients with bvFTD and healthy controls. Cortical thickness and subcortical volume derived from MRI scans were correlated with speech outcomes in patients with bvFTD.

Results: Speech of affected individuals was significantly different from that of healthy controls. The speech signature of patients with bvFTD is characterized by a reduced rate (75\%) and accuracy $(65 \%)$ on alternating syllable production tasks, and prosodic deficits including reduced speech rate (45\%), prolonged intervals (54\%), and use of short phrases (41\%). Groups differed on acoustic measures derived from the reading, unprepared monologue, and diadochokinetic tasks but not the days of the week or sustained vowel tasks. Variability of silence length was associated with cortical thickness of the inferior frontal gyrus and insula and speech rate with the precentral gyrus.

Conclusions: One in 8 patients presented with moderate speech timing deficits with a further twothirds rated as mild or subclinical. Subtle but measurable deficits in prosody are common in bvFTD and should be considered during disease management. Language function correlated with speech timing measures derived from the unprepared monologue only. Neurology ${ }^{\circledR}$ 2017;89:837-844

\section{GLOSSARY}

ALS = amyotrophic lateral sclerosis; AMR = alternating motion rate; bvFTD = behavioral variant of frontotemporal dementia; CBD = corticobasal degeneration; $\mathbf{C l}=$ confidence interval; DDK = diadochokinesis; $\mathbf{F T D}=$ frontotemporal dementia; IvPPA = logopenic variant primary progressive aphasia; nvPPA = nonfluent variant primary progressive aphasia; PALS = Progressive Aphasia Language Scale; PPA = primary progressive aphasia; $\mathbf{P S P}=$ progressive supranuclear palsy; $\mathbf{R O I}=$ region of interest; $\mathbf{S M R}=$ sequential motion rate; svPPA = semantic variant primary progressive aphasia.

The behavioral variant of frontotemporal dementia (bvFTD) is a neurodegenerative disorder representing approximately half of the frontotemporal lobar degenerative disorders. ${ }^{1}$ bvFTD is characterized by disinhibition, apathy, perseverative or compulsive behaviors, hyperorality, functional disability, and executive deficits with relative sparing of memory and visuospatial functions. ${ }^{2}$ Motor symptoms are not considered core characteristics of bvFTD, yet around 20\% of cases using the latest diagnostic criteria present with motor deficits (e.g., parkinsonism, apraxia, gait disturbance). ${ }^{3}$ These findings have led some to suggest that motor symptoms should be included as additional features in the International Consensus Criteria for bvFTD ${ }^{4}$; however, changes in cognition and personality remain central. ${ }^{5}$ Alterations to speech output are also common in bvFTD, with near $60 \%$ of diagnosed cases presenting with deficits ranging from perseveration to mutism, ${ }^{2}$ yet few studies have examined this closely. ${ }^{6}$

Typically communication impairments in bvFTD are thought to be associated with deficits in naming and single word comprehension, and diminished spontaneous speech, ${ }^{7}$ rather than

From the Centre for Neuroscience of Speech (A.P.V., M.L.P., M.W.J.C.) and Florey Institute of Neuroscience and Mental Health (H.P., F.M.C.B., D.D.), University of Melbourne, Australia; Department of Neurodegeneration (A.P.V.), Hertie Institute for Clinical Brain Research, University of Tübingen, Germany; Eastern Cognitive Disorders Clinic (A.P.V., M.L.P., D.D., A.B.), Box Hill Hospital, Monash University; Redenlab Pty. Ltd. (A.P.V., E.L.); and Neuroscience Research Unit (E.L.), Department of Neurology, Sunshine Hospital, Western Health, Melbourne, Australia. Go to Neurology.org for full disclosures. Funding information and disclosures deemed relevant by the authors, if any, are provided at the end of the article. 
overt motor speech impairment, with motor speech deficits usually considered a core diagnostic feature of another frontotemporal dementia (FTD) syndrome, nonfluent variant primary progressive aphasia (nvPPA). ${ }^{8}$ Neuropathologically, bvFTD is characterized by both frontotemporal-parietal cortical atrophy and changes in white matter tract integrity connecting these regions. ${ }^{910}$ The disease has its genesis in the anterior and basal aspects of the frontal lobe, spreading further into the frontal and temporal lobes and subcortex, eventually involving cortical, brainstem, and spinal motor regions. ${ }^{11}$

The overlapping symptomatology of bvFTD in some patients with clinical syndromes associated with corticobasal degeneration $(\mathrm{CBD}),{ }^{12}$ progressive supranuclear palsy (PSP), ${ }^{13}$ and amyotrophic lateral sclerosis $(\mathrm{ALS})^{14}$ can make initial diagnosis difficult. ${ }^{15}$ The FTD-motor neuron disease continuum represents a clear example where there is often clinical heterogeneity and variability in disease trajectory given the presence of cognitive, behavioral, and motor deficits. ${ }^{11}$ The absence of definitive biomarkers prior to postmortem confirmation, and the phenotypic overlap between multiple disorders, means ongoing refinement of the phenotype may improve diagnostic accuracy, capacity to monitor disease progression, and identify targets for intervention.

METHODS Participants. Twenty-four patients (mean age 63.2 years [SD 8.6], 10 male) diagnosed with bvFTD according to established criteria ${ }^{2}$ were recruited from the Eastern Cognitive Disorders Clinic, Melbourne, Australia. Motor characteristics of other clinical syndromes were described separately. We used the clinical diagnosis as our starting point, then checked the clinical features of each patient against the published criteria. ${ }^{2}$ Only patients with a clinical diagnosis of bvFTD who fulfilled the published criteria for probable (not possible) or definite (for those with a known gene mutation) were included in this study. No patients with a motor disorder (e.g., CBD, PSP, ALS) were included in the study. Patients were compared with 24 age- and sex-matched healthy controls (mean age 60.2 years [SD 9.9], 10 male). Mean age at onset was 58.2 years (SD 6.8) and years since diagnosis was 5.0 (SD 3.52) for patients with bvFTD. All controls were native speakers of English and had perceptually normal speech, as judged by 2 speech-language pathologists. No differences in age were observed between groups $(p>0.69)$.

Standard protocol approvals, registrations, and patient consents. Written informed consent was obtained from all participants or their legal guardians. The study was approved by the Human Research Ethics Committee of The University of Melbourne and Eastern Health, Australia.
Speech and language assessments. Language was assessed using the Progressive Aphasia Language Scale (PALS) ${ }^{16}$ and 3 verbal fluency tasks (1, animals; 2 , letters; 3 , alternating between furniture and fruit). Speech was assessed by eliciting 5 different speech samples designed to reflect varying levels of automaticity and complexity. ${ }^{17}$ In reverse order of automaticity, tasks included (1) a monologue for a minimum of 40 seconds within a prespecified semantic category (i.e., tell a story with positive content or happy memory); (2) reading a phonetically balanced paragraph, the Grandfather passage (169 syllables); (3) saying the days of the week; (4) sequential motion rate (SMR) and alternating motion rate (AMR) tasks (i.e., papa or pataka); and (5) sustained vowel /a:/. The monologue is an unprepared task requiring simultaneous novel language formulation and speech production, arguably making it the most cognitively demanding task of the 5 tasks. The reading task does not require novel language formulation; however, it does educe cognitive processes beyond recollection of simple phonetic structures, such as those produced during the SMR task. The days of the week task was designed as an automated stimulus that was identical for all groups, yet did not rely on reading ability. The AMR/SMR tasks were elicited with the instruction "say /stimuli/ repeatedly for 10 seconds as quickly and clearly as possible." The reading, automated, and SMR/AMR tasks were elicited twice to mitigate the effect of unfamiliarity or anxiety associated with experimental testing conditions. ${ }^{18}$ These tasks are stable following a practice trial and sensitive to change and impairment ${ }^{19}$ (see table 1 for analysis details).

Samples were recorded using a Marantz PMD671 solid state recorder coupled with an AKG C520 cardioid head-mounted (frequency range, 20-20 KHz; sensitivity, $-43 \mathrm{~dB}$ ) condenser microphone positioned at a $45^{\circ}$ angle $8 \mathrm{~cm}$ from the mouth. Recordings were sampled at $44.1 \mathrm{KHz}$ and quantized at 8 bits. Speech was quantified via acoustic analysis. Acoustic measures and corresponding tasks are detailed in table 1. A logarithmic natural transformation was applied to all acoustic variables except harmonics to noise ratio to normalize distributions enabling the use of parametric statistical tests. Perceptual characteristics were rated by 2 speech pathologists blinded to diagnosis using consensus ratings. Severity was rated on a scale of $0-4$ where $0=$ no impairment, 1 = subclinical impairment (perceivable but not clinical), $2=$ mild, $3=$ moderate, and $4=$ severe impairment.

MRI data acquisition and analysis. 3D isotropic T1weighted magnetization-prepared rapid gradient echo scans were acquired on a clinical Siemens (Munich, Germany) Aera 1.5T scanner (Eastern Health, Australia). The parameters applied were as follows: flip angle $=15^{\circ}$, field of view $=256 \times 256$, repetition time $=1,900 \mathrm{~ms}$, echo time $=2.67 \mathrm{~ms}$, matrix size $=$ $256 \times 256$ pixels, slice thickness $=1 \mathrm{~mm}$, number of slices $=$ 144. Data analyses were conducted using Freesurfer version 5.1.3 using standard defaults (surfer.nmr.mgh.harvard.edu). Freesurfer processing includes correction for magnetic field inhomogeneities and skull stripping to remove nonbrain tissue. Segmentation of gray and white matter structures was reconstructed to measure thickness at each surface location, using a semiautomated approach..$^{20}$ Regions of interest (ROIs) were parcellated according to procedures outlined by Freesurfer. ${ }^{21}$ ROI masks were inspected following semiautomated classification and manual edits were made where needed. Whole brain volume was determined via intracranial volume and global measures of cortical gray and white matter were obtained. Volumetric measures (average gray matter thickness) were created for left and right insulae, pars opercularis, precentral gyrus, and caudate nucleus. ROIs were identified as salient sites for speech motor control based on primary progressive aphasia (PPA)/bvFTD imaging data. ${ }^{22-24}$ 
Table 1 Acoustic and perceptual analysis measures

\begin{tabular}{|c|c|c|c|}
\hline Measure & Purpose & Calculation & Stimuli \\
\hline \multicolumn{4}{|l|}{ Acoustic } \\
\hline \multirow{3}{*}{$\begin{array}{l}\text { Mean silence length, variation of } \\
\text { silence length, percent silence }{ }^{40} ; \\
\text { calculated in Praat (software } \\
\text { program) using automated scripts }\end{array}$} & Global measures of timing & Derived from intensity contour & $\begin{array}{l}\text { Monologue, reading, automated } \\
\text { tasks }\end{array}$ \\
\hline & & $\begin{array}{l}\text { Mean silence length: number of silences/ } \\
\text { total silence duration (average silence } \\
\text { length) }\end{array}$ & \\
\hline & & $\begin{array}{l}\text { Percent silence: total speech time/total } \\
\text { silence time } \times 100 \text {; silences removed } \\
\text { from start and end of samples prior to } \\
\text { analysis. }\end{array}$ & \\
\hline Speech rate & & $\begin{array}{l}\text { Speech rate: number of syllables/ } \\
\text { duration of sample }\end{array}$ & Reading and automated tasks \\
\hline Harmonics to noise ratio & Voice quality & $\begin{array}{l}\text { Quantifies the amount of additive noise } \\
\text { in the voice }\end{array}$ & Sustained vowel \\
\hline fo coefficient of variation & Vocal control & $\begin{array}{l}\text { Quantifies variance of fundamental } \\
\text { frequency independent of speaker sex }\end{array}$ & Sustained vowel \\
\hline \multicolumn{4}{|l|}{ Perceptual } \\
\hline Voice quality & Laryngeal function & & Sustained vowel, monologue \\
\hline Resonance & Velopharyngeal function & & Monologue \\
\hline Phonemic and phonetic errors & Language vs speech errors & & Reading, monologue \\
\hline Prosody (rate, stress) & $\begin{array}{l}\text { Naturalness; refers to the degree to } \\
\text { which speech sounds like that of } \\
\text { healthy, native speakers }\end{array}$ & & Monologue \\
\hline
\end{tabular}

Abbreviations: $\mathrm{AMR}=$ alternating motion rate; $\mathrm{SMR}=$ sequential motion rate.

Statistical analysis. Differences between groups were explored using parametric and nonparametric methods. Speech acoustic data were compared via independent sample $t$ tests. Language and speech outcomes derived perceptually were compared using nonparametric equivalents (MannWhitney $U$ ). Spearman $\rho$ was used to examine correlations between language outcomes on the PALS and verbal fluency with acoustic speech outcomes that significantly differed between healthy controls and bvFTD. Correlations between ROIs and acoustic speech outcomes that significantly differed between healthy controls and bvFTD were calculated using Spearman $\rho$ with an adjusted significance level of $p<0.005$ to cater for multiple comparisons.

RESULTS Language. Significance was adjusted for multiple comparisons $(p<0.005)$. Differences between groups were observed on the single word comprehension domain of the PALS $(U=3.51$, $p<0.001)$ and the 2 semantic verbal fluency tasks (category: $U=-3.45, p<0.001$; alternating: $U=$ -3.9, $p<0.001$ ) (table e-1 at Neurology.org). No other language domains differed between groups. Associations were observed between mean silence length and silence length variability on the free speech task and performance on the naming $(p<0.001)$ and sentence comprehension domains of PALS $(p=0$. 002) as well as all 3 verbal fluency tasks (category: $p<$ 0.001; letter: $p=0.003$; alternating: $p<0.001$ ). Proportion of silence on the free speech task was correlated with category and alternating verbal fluency tasks ( $p=0.001$ and $p<0.001$ respectively). No other significant correlations were observed between speech and language measures.

Subjective: Listener-based analysis of speech. Significance was adjusted for multiple comparisons $(p<$ $0.005)$. A total of $75 \%$ ( $95 \%$ confidence interval [CI] $58 \%-92 \%)$ of patients presented with some form of speech deficit compared to age- and sex-matched controls, demonstrating that speech impairment is a very common feature of bvFTD. Four of the 26 perceptual measures differed (i.e., $p<0.005$ ) between patients and controls: speech rate $(p=0.002)$, use of short phrases rather than longer complex sentences $(p=0.002)$, prolonged intervals $(p<0.001)$, and speed during diadochokinesis (DDK) $(p=0.003)$. A further 9 measures did not differ between groups following adjustment for 
multiple comparisons: variable rate ( $p=0.037$ ), reduced stress $(p=0.007)$, equal and excess stress $(p=0.039)$, strain-strangled voice quality $(p=0.009)$, imprecise consonants $(p=0.01)$, prolonged phonemes $(p=$ 0.039 ), phonemic errors $(p=0.01)$, groping ( $p=$ $0.039)$, and regularity of $\operatorname{DDK}(p=0.007)$ (table 2$)$.

Objective: Acoustic analysis of speech. When adjusted for multiple comparisons (set at $p<0.005$ ), differences were observed between groups on 11/19 acoustic measures including the reading task (percent silence, speech rate), the unprepared monologue task (mean pause length, variability of silence length, percent silence), the AMR task (average rate, mean period length), and the SMR task (average rate, mean period length) (table 3). No differences were observed between groups on the remaining acoustic measures including fundamental frequency, voice quality, or

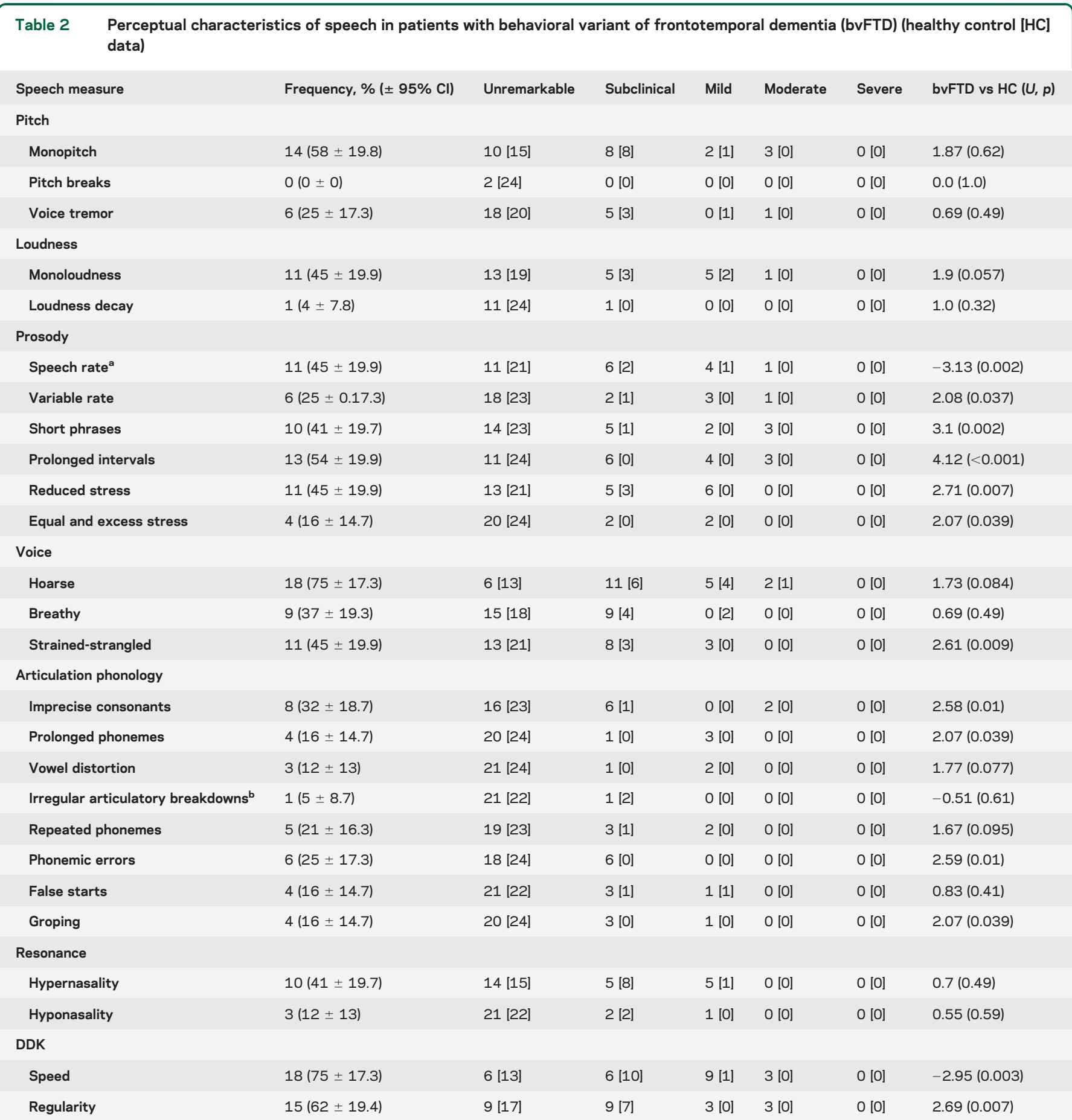

Abbreviations: $\mathrm{Cl}=95 \%$ confidence interval; $\mathrm{DDK}=$ diadochokinesis; $U=$ independent samples Mann-Whitney $U$ test converted to standardized $t$ statistic ( $p$ value).

${ }^{\text {a }}$ Ratings of severity refer to reduced rate of speech: 2 patients presented with subclinical increased speech rate.

${ }^{\mathrm{b}}$ Missing data from 2 participants preventing adequate evaluation of metric. 
Table 3 Acoustic profile of patients with behavioral variant of frontotemporal dementia (bvFTD) and healthy controls

\begin{tabular}{|c|c|c|c|c|}
\hline Task & Acoustic measure & \multicolumn{2}{|l|}{ Mean \pm SD } & $\frac{t \text { Test }}{t(p)}$ \\
\hline \multirow[t]{4}{*}{ Days of the week } & Mean silence ${ }^{a}$ & $0.093 \pm 0.051$ & $0.067 \pm 0.038$ & $-2.01(0.05)$ \\
\hline & Variability of silence length ${ }^{b}$ & $0.058 \pm 0.046$ & $0.041 \pm 0.043$ & $-1.55(0.13)$ \\
\hline & Proportion of silence time ${ }^{c}$ & $12.72 \pm 6.56$ & $12.46 \pm 6.07$ & $0.26(0.8)$ \\
\hline & Speech rate ${ }^{d}$ & $3.8 \pm 1.2$ & $4.12 \pm 1.07$ & $1.01(0.32)$ \\
\hline \multirow{3}{*}{ Reading passage } & Variability of silence length ${ }^{b}$ & $0.27 \pm 0.14$ & $0.19 \pm 0.07$ & $-2.79(0.008)$ \\
\hline & Proportion of silence time ${ }^{c}$ & $28.54 \pm 8.44$ & $22.47 \pm 5.75$ & $-3.01(0.004)$ \\
\hline & Speech rate ${ }^{d}$ & $3.07 \pm 0.82$ & $3.9 \pm 0.53$ & $4.38(<0.001)$ \\
\hline Unprepared monologue & Mean silence ${ }^{a}$ & $0.35 \pm 0.22$ & $0.15 \pm 0.06$ & $-5.83(<0.001)$ \\
\hline \multirow{2}{*}{ SMR } & Mean DDK rate & $4.36 \pm 1.79$ & $6.38 \pm 0.88$ & $4.88(<0.001)$ \\
\hline & Covariance DDK period & $37.54 \pm 16.96$ & $28.49 \pm 15.54$ & $-1.91(0.063)$ \\
\hline \multirow[t]{3}{*}{ AMR } & Mean DDK period & $255.72 \pm 82.68$ & $162.69 \pm 15.2$ & $-4.17(<0.001)$ \\
\hline & Mean DDK rate & $4.87 \pm 1.33$ & $6.19 \pm 0.54$ & $4.7(<0.001)$ \\
\hline & Covariance of DDK period & $20.96 \pm 29.51$ & $9.45 \pm 4.54$ & $-1.59(0.12)$ \\
\hline \multirow[t]{2}{*}{ Sustained vowel } & Harmonics to noise ratio & $16.99 \pm 6.23$ & $19.93 \pm 8.3$ & $1.349(0.18)$ \\
\hline & fo CoV & $0.067 \pm 0.061$ & $0.066 \pm 0.08$ & $-0.81(0.42)$ \\
\hline
\end{tabular}

Abbreviations: $A M R=$ alternating motion rate (e.g., pataka); $\mathrm{CoV}=$ coefficient of variation; DDK = diadochokinetic rate; fO = fundamental frequency; $\mathrm{SMR}=$ sequential motion rate (e.g., papapa).

The $f O$ varies depending on sex of speaker and was therefore not included. CoV of $f O$ is a measure of variation around the mean and thus not dependent on sex of speaker.

${ }^{a}$ Mean silence length (determined acoustically) was correlated with prolonged intervals (determined perceptually) on the monologue $(\rho=0.57, p=0.004)$ but not the days of the week task ( $\rho=-0.14, p=0.52$ ) or the reading passage $(\rho=0.38, p=0.07)$.

${ }^{b}$ Variability of silence length (determined acoustically) was not correlated with variable rate (determined perceptually) on any task (days of the week: $\rho=$ $0.08, p=0.74$; reading passage: $\rho=0.25, p=0.26$; unprepared monologue: $\rho=0.44, p=0.03$ ).

${ }^{\mathrm{c}}$ Proportion of silence time (determined acoustically) was correlated with prolonged intervals (determined perceptually) on the unprepared monologue ( $\rho=$ $0.63, p=0.001)$ but not the days of the week task $(\rho=0.14, p=0.53)$ or the reading passage $(\rho=0.4, p=0.06)$.

${ }^{d}$ Speech rate (determined acoustically) was correlated with speech rate (determined perceptually) on the reading passage $(\rho=0.651, p=0.001)$ but not on the days of the week task $(\rho=0.497, p=0.013)$.

timing measures derived from the days of the week task.

Correlation between acoustic measures of speech and MRI ROIs. Only those acoustic speech measures differing significantly between groups were examined. Significance was adjusted for multiple comparisons $(p<0.005)$. The size of correlations between some measures of speech timing derived from the reading task and ROIs were meaningful with large effect sizes (where small, medium, and large effects are $r=0.1$, $0.3,0.5$, respectively ${ }^{25}$ ); however, they were not statistically significant following adjustment for multiple comparisons. Large correlation coefficients were observed: silence length variability and cortical thickness of the left pars triangularis $(\rho=0.551, p=$ 0.032 ), right pars opercularis ( $\rho=0.571, p=$ $0.017)$, right pars orbitalis $(\rho=0.636, p=0.011)$, and the right insula $(\rho=0.517, p=0.034)$; percent silence and thickness of the right pars orbitalis $(\rho=$ $0.654, p=0.008)$ and volume of the right cerebellum $(\rho=-0.522, p=0.032)$; and speech rate with thickness of the left caudal anterior cingulate $(\rho=$ $-0.551, p=0.022)$ and right precentral gyrus $(\rho=$ $0.544, p=0.024)$.

DISCUSSION Motor speech impairments are described as an additional feature of bvFTD by some, ${ }^{4}$ yet there is no systematic or objective evidence on their nature and severity. We present a comprehensive description of motor speech function in bvFTD. Objective and listener-based comparisons showed differences between the motor speech profile of patients with bvFTD and age-/sex-matched healthy controls. Three-quarters of patients with bvFTD presented with speech deviating from healthy controls on select tasks. The speech signature of bvFTD is 
characterized by short phrases (rather than longer complex sentences) and variable and prolonged intervals between words, resulting in reduced rate during conversation, reduced stress, and slower rate and regularity during DDK tasks. Some of these prosodic deficits were observed in 75\% (95\% CI $58 \%-92 \%)$ of patients, half with subtle impairments and half rated as mild or moderate severity. A smaller proportion of patients also presented with subtle (subclinical) evidence of strain-strangled voice quality (45\%), imprecise consonants (32\%), and phonemic errors $(25 \%)$, deviating from controls. Patients with bvFTD performed worse on single word comprehension and verbal fluency tasks compared to controls, similar to earlier reports. ${ }^{7}$

These deficits combine to produce speech distinct from healthy speakers and other PPAs (see supplementary audio files $1-3$ for prototypical examples of speech of patients with bvFTD). The speech signature of bvFTD does not suggest a profile of apraxia of speech common in nvPPA or halting production as severe as in semantic variant PPA (svPPA) or logopenic variant PPA (lvPPA) but a timing-based deficit in the absence of other overt signs of dysarthria such as clinically observable slurred speech. These data in part support earlier work describing patients with bvFTD producing speech at a reduced rate with shorter phrases on a picture description task compared to healthy speakers. ${ }^{26}$

All FTD and PPA syndromes result in a slower rate of speech. ${ }^{6}$ Syndromes differ because of the presence or absence of language or apraxia-based speech deficits. In svPPA and lvPPA, a slow rate of speech can result from word-finding difficulties, ${ }^{27,28}$ whereas slow or halting speech in nvPPA can arise from agrammatism, apraxia of speech, and oral apraxia. ${ }^{28}$ The speech profile of lvPPA is dominated by phonologic sound substitutions (phonemic paraphasias) in the absence of motor speech errors ${ }^{6,28}$ and individuals with nvPPA can present with consonant and vowel distortions, groping, prolonged phonemes, and excess and equal stress. ${ }^{29}$ In line with the diagnostic criteria for bvFTD, the reduced rate of speech observed in patients with bvFTD compared to controls could arise from deficits in executive functioning rather than overt issues relating to speech motor planning or semantic/lexical retrieval. If timing data from reading and monologue tasks were taken alone, the speech of patients with bvFTD appears to be the result of cognitive decline. However, evidence of mild to moderate deficits demonstrated in less cognitively demanding tasks such as DDK suggest an underlying motor timing deficit affecting speech in conjunction with existing cognitive decline.

No correlations survived adjustment for multiple comparisons and these data need to be interpreted with caution. Large correlation coefficients (i.e., $\rho=0.52-0.66, p<0.05)$ were observed between measures of timing in areas traditionally associated with speech. Variability of silence length correlated with cortical thickness of the inferior frontal gyrus and insula and speech rate with the right precentral gyrus. These regions have been repeatedly cited as critical sites for articulatory planning, ${ }^{30}$ speech fluency, ${ }^{31}$ and coordination of muscles engaged in phonation and articulation. ${ }^{32}$ Surprisingly, performance on the DDK task correlated with average thickness of the temporal poles bilaterally. The DDK task requires the rapid repetition of sequentially changing syllables (i.e., pataka) as quickly and as clearly as possible and difficulties completing this task are thought to reflect impairment in speech planning or execution. Functional imaging work suggests this region may be involved in semantic processing ${ }^{33}$ with involvement of the temporal poles in a broader language network. $^{34}$ The mild deficits in speech sequencing observed in this study may be the result of deterioration of high-level cognitive control and attentional processes, ${ }^{35}$ possibly linked to decreased cortical connectivity within the salience network. ${ }^{36}$ However, the link here may not be causative but concurrent, given the role temporal poles play in regulating emotion processing in behavioral disorders. ${ }^{37}$

We observed that some speech tasks and acoustic measures (such as timing metrics derived from the monologue, reading, DDK) were sensitive to speaker pathology, whereas others were disordered in both patients with bvFTD and healthy controls (e.g., hoarse voice, $75 \%$; monopitch, $38 \%$ ). If as suggested, speech tasks fit along a continuum of automaticity, ${ }^{17}$ those tasks with increased cognitive load (e.g., unprepared monologue) should be more adversely affected by cognitive disorders where a motor impairment is not anticipated. This hypothesis is supported here, with the only statistically significant relationship observed between language domains and measures of speech timing derived from the monologue task (theorized to be the most cognitively demanding task). No relationship was observed between performance on language tests and the DDK task. Here we found differences between groups on both connected speech tasks with low levels of automaticity (reading, monologue) and speech tasks primarily designed to examine motor speech function with less demand on language formulation (DDK), suggesting a combination of both cognitive and motor impairment in patients with bvFTD. The motor demands of the DDK task (i.e., coordinating 3 subsystems of speech [respiration, phonation, and articulation] repetitively while changing place of articulation [lips, alveolar, back]) can be challenging in patients with neuromuscular disorders and are often used in oral motor 
examinations and disease severity scores. Impaired performance on DDK tasks is not, however, typically associated with behavioral disorders such as dementia, where deficits on the task may be the result of impaired attention or reduced motivation. It is possible that aspects of bvFTD play a part in this experimental context. Similarly, the novelty of DDK tasks may result in altered production, as speakers unfamiliar with the task may produce speech differently from controls. The effect of task novelty, however, was thought to be mitigated by eliciting a practice of the protocol prior to analysis. ${ }^{19}$ Further, if attention or motivation were the reasons behind DDK deficits observed here, similar differences between groups may be found on other simple tasks, such as days of the week, which is brief, does not require reading, and is potentially likewise demotivating for the speaker.

Objective measures of speech that utilize continuous variables can assist in quantifying the degree and nature of deficits, with particular sensitivity for measuring change over time or in response to treatment. ${ }^{18}$ With a few exceptions, ${ }^{23}$ acoustic analysis and subsequent objective differentiation of bvFTD and PPA speech profiles is not widely pursued as characterization in the dementias continues to rely on subjective clinical judgment. This may be mainly for practical reasons, as examiners tend to make clinician-based judgments, sometimes strengthened by semiquantitative analysis of connected speech (e.g., error rates, syntactic complexity, lexical and semantic content ${ }^{38,39}$ ). Findings from this study demonstrate the utility of combining objective and listener-based judgements of speech by describing the speech signature of bvFTD.

\section{AUTHOR CONTRIBUTIONS}

Adam Vogel: study concept and design, acquisition of data, analysis or interpretation of data, drafting/revising the manuscript, study supervision. Matthew Poole: acquisition of data, analysis or interpretation of data, critical revision of the manuscript for intellectual content. Hugh Pemberton: acquisition of data, analysis or interpretation of data, critical revision of the manuscript for intellectual content. Marja Caverlé: analysis or interpretation of data, critical revision of the manuscript for intellectual content. Frederique Boonstra: analysis or interpretation of data, critical revision of the manuscript for intellectual content. Essie Low: acquisition of data, critical revision of the manuscript for intellectual content. David Darby: acquisition of data, analysis or interpretation of data, critical revision of the manuscript for intellectual content. Amy Brodtmann: acquisition of data, analysis or interpretation of data, critical revision of the manuscript for intellectual content, study supervision.

\section{STUDY FUNDING}

No targeted funding reported.

\section{DISCLOSURE}

A. Vogel is funded by an NHMRC grant (1082910) and Australian Research Council grants (LP130100026, DP130101900) and serves on the editorial board for the International Journal of Speech-Language Pathology and the journal Speech, Language, Hearing. M. Poole, H. Pemberton, M. Caverlé, F. Boonstra, E. Low, and D. Darby report no disclosures relevant to the manuscript. A. Brodtmann serves on the scientific advisory board of the Brain Health Initiative, a US-based firm, and serves on the editorial board for Neurology ${ }^{\circledR}$ and the International Journal of Stroke. Go to Neurology.org for full disclosures.

Received November 23, 2016. Accepted in final form May 30, 2017.

\section{REFERENCES}

1. Ratnavalli E, Brayne C, Dawson K, Hodges JR. The prevalence of frontotemporal dementia. Neurology 2002;58: 1615-1621.

2. Rascovsky K, Hodges JR, Knopman D, et al. Sensitivity of revised diagnostic criteria for the behavioural variant of frontotemporal dementia. Brain 2011;134: 2456-2477.

3. Chare L, Hodges JR, Leyton CE, et al. New criteria for frontotemporal dementia syndromes: clinical and pathological diagnostic implications. Neurosurg Psychiatry 2014;85:865-870.

4. Piguet O, Hornberger M, Mioshi E, Hodges JR. Behavioural-variant frontotemporal dementia: diagnosis, clinical staging, and management. Lancet Neurol 2011;10: $162-172$.

5. Rascovsky K, Grossman M. Clinical diagnostic criteria and classification controversies in frontotemporal lobar degeneration. Int Rev Psychiatry 2013;25:145-158.

6. Poole ML, Brodtmann A, Darby D, Vogel AP. Motor speech phenotypes of frontotemporal dementia, primary progressive aphasia, and progressive apraxia of speech. J Speech Lang Hear Res 2017;60:897-911.

7. Hardy CJ, Buckley AH, Downey LE, et al. The language profile of behavioral variant frontotemporal dementia. J Alzheimer's Dis 2016;50:359-371.

8. Gorno-Tempini ML, Hillis AE, Weintraub S, et al. Classification of primary progressive aphasia and its variants. Neurology 2011;76:1006-1014.

9. Whitwell JL, Avula R, Senjem ML, et al. Gray and white matter water diffusion in the syndromic variants of frontotemporal dementia. Neurology 2010;74:1279-1287.

10. Mahoney CJ, Ridgway GR, Malone IB, et al. Profiles of white matter tract pathology in frontotemporal dementia. Hum Brain Mapp 2014;35:4163-4179.

11. Burrell JR, Halliday GM, Kril JJ, et al. The frontotemporal dementia-motor neuron disease continuum. Lancet 2016;388:919-931.

12. Kertesz A, Martinez-Lage P, Davidson W, Munoz DG. The corticobasal degeneration syndrome overlaps progressive aphasia and frontotemporal dementia. Neurology 2000;55:1368-1375.

13. Boeve BF, Lang AE, Litvan I. Corticobasal degeneration and its relationship to progressive supranuclear palsy and frontotemporal dementia. Ann Neurol 2003;54:S15-S19.

14. Lomen-Hoerth C, Anderson T, Miller BL. The overlap of amyotrophic lateral sclerosis and frontotemporal dementia. Neurology 2002;59:1077-1079.

15. Woolley JD, Khan BK, Murthy NK, Miller BL, Rankin $\mathrm{KP}$. The diagnostic challenge of psychiatric symptoms in neurodegenerative disease: rates of and risk factors for prior psychiatric diagnosis in patients with early neurodegenerative disease. J Clin Psychiatry 2011;72: 126-133.

16. Leyton CE, Villemagne VL, Savage S, et al. Subtypes of progressive aphasia: application of the international consensus criteria and validation using $\beta$-amyloid imaging. Brain 2011;134:3030-3043. 
17. Vogel AP, Fletcher J, Maruff P. The impact of task automaticity on speech in noise. Speech Commun 2014;65:1-8.

18. Vogel AP, Maruff P. Monitoring change requires a rethink of assessment practices in voice and speech. Logoped Phoniatr Vocol 2014;39:56-61

19. Vogel AP, Fletcher J, Snyder PJ, Fredrickson A, Maruff P. Reliability, stability, and sensitivity to change and impairment in acoustic measures of timing and frequency. J Voice 2011;25:137-149.

20. Fischl B. FreeSurfer. NeuroImage 2012;62:774-781.

21. Fischl B, Salat DH, Busa E, et al. Whole brain segmentation: automated labeling of neuroanatomical structures in the human brain. Neuron 2002;33:341-355.

22. Whitwell JL, Duffy JR, Strand EA, et al. Neuroimaging comparison of primary progressive apraxia of speech and progressive supranuclear palsy. Eur J Neurol 2013;20: 629-637.

23. Ballard KJ, Savage S, Leyton CE, Vogel AP, Hornberger M, Hodges JR. Logopenic and nonfluent variants of primary progressive aphasia are differentiated by acoustic measures of speech production. PLoS One 2014;9:e89864.

24. Rohrer JD, Caso F, Mahoney C, et al. Patterns of longitudinal brain atrophy in the logopenic variant of primary progressive aphasia. Brain Lang 2013;127:121-126.

25. Cohen J. Statistical Power Analysis for the Behavioral Sciences. 2nd ed. Hillsdale, NJ: Lawrence Erlbaum; 1988.

26. Ash S, Evans E, O'Shea J, et al. Differentiating primary progressive aphasias in a brief sample of connected speech. Neurology 2013;81:329-336.

27. Ash S, Moore P, Vesely L, et al. Non-fluent speech in frontotemporal lobar degeneration. J Neurolinguistics 2009;22:370-383.

28. Croot K, Ballard KJ, Leyton CE, Hodges JR. Apraxia of speech and phonological errors in the diagnosis of nonfluent/agrammatic and logopenic variants of primary progressive aphasia. J Speech Lang Hear Res 2012;55: S1562-S1572.

29. Ogar JM, Dronkers NF, Brambati SM, Miller BL, GornoTempini ML. Progressive nonfluent aphasia and its char- acteristic motor speech deficits. Alzheimer Dis Assoc Disord 2007;21:S23-S30.

30. Price CJ. The anatomy of language: a review of $100 \mathrm{fMRI}$ studies published in 2009. Ann NY Acad Sci 2010;1191: 62-88.

31. Fridriksson J, Guo D, Fillmore P, Holland A, Rorden C. Damage to the anterior arcuate fasciculus predicts nonfluent speech production in aphasia. Brain 2013;136: 3451-3460.

32. Ackermann H, Riecker A. The contribution of the insula to motor aspects of speech production: a review and a hypothesis. Brain Lang 2004;89:320-328.

33. Patterson K, Nestor PJ, Rogers TT. Where do you know what you know? The representation of semantic knowledge in the human brain. Nat Rev Neurosci 2007;8: 976-987.

34. Geranmayeh F, Wise RJS, Mehta A, Leech R. Overlapping networks engaged during spoken language production and its cognitive control. J Neurosci 2014;34: 8728-8740.

35. Menon V, Uddin LQ. Saliency, switching, attention and control: a network model of insula function. Brain Struct Funct 2010;214:655-667.

36. Filippi M, Agosta F, Scola E, et al. Functional network connectivity in the behavioral variant of frontotemporal dementia. Cortex 2013;49:2389-2401.

37. Alegria AA, Radua J, Rubia K. Meta-analysis of fMRI studies of disruptive behavior disorders. Am J Psychiatry 2016;173:1119-1130.

38. Ahmed S, Haigh AMF, de Jager CA, Garrard P. Connected speech as a marker of disease progression in autopsy-proven Alzheimer's disease. Brain 2013;136: 3727-3737.

39. Wilson SM, Henry ML, Besbris M, et al. Connected speech production in three variants of primary progressive aphasia. Brain 2010;133:2069-2088.

40. Rosen K, Murdoch B, Folker J, et al. Automatic method of pause measurement for normal and dysarthric speech. Clin Linguistics Phonetics 2010;24:141-154.

\section{Get 10 AAN Practice Management Webinars for Less than \$19 Each}

The AAN is dedicated to helping neurologists improve their practices and delivery of quality care to their patients. Our popular Practice Management Webinars offer the latest expert information and insights to help you navigate through the changes that lay ahead in the new health care landscape. And they offer the flexibility to attend live webinars or access them on demand at a more convenient time.

Purchase webinars individually for $\$ 99$ each, or subscribe to the complete series of 2017 webinars for only $\$ 189$ - that's less than $\$ 19$ per webinar! See the list of webinars and subscribe at $A A N . c o m / v i e w / p m w 17$. 